\title{
Survey of integrated statistical computer packages
}

\author{
RONALD A. BERK \\ Division of Education, Johns Hopkins University, Baltimore, Maryland 21218
}

The statistical features and general characteristics of seven integrated packages were surveyed. SPSS, DATA-TEXT, and OSIRIS emerged as the most comprehensive. OSIRIS in particular offered the most extensive multivariate analysis capabilities. The only category of statistical analysis neglected in all of the packages was nonparametric procedures related to ordinal data. Information on availability and user's manuals is provided.

Several recent surveys of statistical computer programs have been conducted (Allerbeck, 1973; Anderson \& Coover, 1972; Armor, 1970; Coover, Dyer, Gross, Johnson, Lutgen, \& Miner, 1974; Schucany, Minton, \& Shannon, 1972; Hall, Note 1, 2; Rowe, Hall, \& Scheer, Note 3; Tauchi, Note 4). Despite the number of programs reviewed, no comprehensive examination of the specific statistical procedures included in the programs has been undertaken. This paper surveys the statistical features and general characteristics of seven popular integrated packages: (1) Statistical Package for the Social Sciences (SPSS), (2) DATA-TEXT, (3) Organized Set of Integrated Routines for Investigation with Statistics (OSIRIS), (4) OMNITAB, (5) Statistical Analysis System (SAS), (6) MANOVA, (7) MULTIVARIANCE (MULT). No attempt has been made to evaluate the computational accuracy of the individual routines. The statistical effectiveness of the packages is currently being assessed by the Committee on Evaluation of Statistical Program Packages, Section on Statistical Computing, American Statistical Association (Francis, Heiberger, \& Velleman, 1975).

\section{METHOD}

The first step in the survey was the compilation of the statistical techniques employed most often by researchers. A list of descriptive, parametric, and nonparametric statistics was developed. 1 It is by no means exhaustive of the numerous methods of data analysis being used.

Nine integrated computer packages were selected based on their frequency of use in descriptive and experimental research and compatibility with third-generation computers. Complete documentation was obtainable for all except P-STAT and SOUPAC. The seven remaining packages were divided into two categories: generalized and specialized. This distinction was made to differentiate between five which offer both data maintenance and statistical analysis capabilities (SPSS, DATA-TEXT, OSIRIS, OMNITAB, SAS) and two that provide only the latter (MANOVA, MULTIVARIANCE).

A thorough examination of the documentation currently available for each package was conducted. All statistics designated as mandatory or optional program output were identified. A statistic that did not satisfy this criterion was not listed for the package, for example, multivariate analysis of variance
(MANOVA) output not designated by the package although obtainable from a multiple regression program.

\section{RESULTS AND DISCUSSION}

The statistical features and general characteristics of the packages are presented in Tables 1 and 2, respectively. SPSS, DATA-TEXT, and OSIRIS appear to offer the most comprehensive analyses for the three statistical categories. OSIRIS distinguishes itself within this group by requiring the least core and providing the most extensive multivariate analysis capabilities. The MANOVA program in OSIRIS is, in fact, an earlier version of the MANOVA package reviewed in this survey (Clyde, Cramer, \& Sherin, 1966). In addition to the capabilities listed in Table 1, OSIRIS includes Automatic and Theta-Aid Interaction Detector programs (AID, THAID) and multivariate nominal, multiple classification, and configuration analyses. Data simulation and lag-lead management programs are also provided. The latter is particularly useful for preparing data for time-series experiments.

Despite the outstanding features of OSIRIS, SPSS has become the most widely used package. It can be found at over 700 installations throughout the world and is the most used package for academic survey analysis in the United Kingdom (Rowe et al., Note 3). Current developmental efforts are focusing on an interactive version, capable of reading and producing batchSPSS-format system files, and Versions 7 and 8 with test and scale item analyses, nonlinear regression tests, log linear modeling, path analysis, frequency and joint frequency distributions of multiple response variables, Automatic Interaction Detection (AID), and a variety of nonparametric tests for ordinal data (SPSS, Inc., Note 5). SPSS, along with OSIRIS and SAS, also contains a Guttman scaling program.

Within the generalized package group, OMNITAB provides an attractively compact and versatile (machineindependent) altemative to the larger packages at a fraction of the cost. It contains the basic descriptive 
Table 1

Statistical Features of Seven Integrated Packages

Integrated Packages

Statistics

SPSS DATA-TX OSIRIS OMNITAB SAS MANOVA MULT

I. Descriptive Statistics
A. Mean
B. Median
C. Mode
D. Range
E. Variance
F. Standard deviation
G. Standard error (mean)
H. Skewness
I. Kurtosis
J. Frequency distribution
K. Crosstabulation
L. Scattergram/plot

$\begin{array}{ll}\mathbf{X} & \mathbf{X} \\ \mathbf{X} & \mathbf{X} \\ \mathbf{X} & \\ \mathbf{X} & \\ \mathbf{X} & \mathbf{X} \\ \mathbf{X} & \mathbf{X} \\ \mathbf{X} & \\ \mathbf{X} & \mathbf{X} \\ \mathbf{X} & \mathbf{X} \\ \mathbf{X} & \mathbf{X} \\ \mathbf{X} & \mathbf{X} \\ \mathbf{X} & \mathbf{X}\end{array}$

$\mathbf{X}$
$\mathbf{X}$
$\mathbf{X}$
$\mathbf{X}$
$\mathbf{X}$

$\mathbf{X}$
$\mathbf{X}$
$\mathbf{X}$
$\mathbf{X}$
$\mathbf{X}$

$\mathbf{X}$

X

$\mathrm{X}$

$\mathbf{X}$

$\mathrm{X} \quad \mathrm{X}$

$\begin{array}{ll}\mathbf{X} & \mathbf{X} \\ \mathbf{X} & \end{array}$

II. Parametric Statistics

A. Correlation

1. Pearson

2. Biserial

3. Tetrachoric

4. Partial

$\mathbf{X}$

$\mathbf{X}$

$\mathbf{X}$

$\mathbf{X}$

B. Univariate Analysis

$\mathrm{X}^{*}$

$\mathrm{X}$

$\mathrm{X}$

$\mathbf{X}$

$\mathbf{X}$

1. t test (independent samples)

2. $t$ test (correlated samples)

3. ANOVA/ANCOVA

4. Factorial ANOVA/ANCOVA

C. Multivariate Analysis

1. MANOVA/MANCOVA

2. Multiple correlation/regression

3. Simple/multiple discriminant analysis

$\mathbf{X}$
$\mathbf{X}$
$\mathbf{X}$
$\mathbf{X}$

4. Canonical correlation

5. Factor analysis

6. Cluster analy sis

7. Multidimensional scaling

$\mathrm{X}$
$\mathrm{X}$
$\mathrm{X}$
$\mathrm{X}^{* *}$

$\mathrm{X}$

$X$
$X^{* *}$

$X_{\dagger}^{\dagger}$
$X_{\dagger}^{\dagger}$

$\mathbf{X}$
$\mathbf{X}^{* *}$
$\mathrm{X}_{+\dagger}$
$\mathbf{X}$
$\mathbf{X}$
$\mathbf{X}$
$\mathbf{X}$

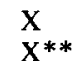

$\mathbf{X}$

$\mathrm{X} * *$

$\begin{array}{ll}\mathbf{X} & \mathbf{X} \\ \mathbf{X} & \mathbf{X} \\ \mathbf{X} & \mathbf{X} \\ \mathbf{X} & \end{array}$

$\begin{array}{ll} & \mathbf{X} \\ \mathbf{X} & \mathbf{X} \\ \mathbf{X} & \\ & \\ \mathbf{X} & \mathbf{X} \\ & \mathbf{X} \\ & \mathbf{X}\end{array}$

III. Nonparametric Statistics
A. Nominal Data
1. Chi-square
2. McNemar's test
3. Fisher's exact test
4. Cochran's Q
5. Contingency coefficient
6. Phi coefficient
7. Cramer's V
8. Goodman-Kruskal's lambda

$\mathrm{X}$

$X$
$X$
$X$

$\mathrm{X}$

$\begin{array}{lll}\mathbf{X}+\dagger & X^{* *} & X^{* *} \\ \mathbf{X} & & \mathbf{X} \\ \mathbf{X} & & \mathbf{X} \\ \mathbf{X} & & \mathbf{X}\end{array}$

B. Ordinal Data

1. Kolmogorov-Smirnov's tests

2. Runs test

3. Sign test

4. Wilcoxon's matched-pairs test

5. Median test

6. Mann-Whitney's U

7. Wald-Wolfowitz's runs test

8. Moses' test of extreme reactions

9. Friedman's ANOVA

10. Kruskal-Wallis' ANOVA

11. Spearman's tho

12. Kendall's tau

13. Kendall's W

14. Goodman-Kruskal's gamma

15. Somer's D

$\begin{array}{lll}\mathbf{X} & \mathbf{X} & \mathbf{X} \\ \mathbf{X} & & \\ \mathbf{X} & \mathbf{X} & \\ \mathbf{X}^{*} & & \mathbf{X} \\ \mathbf{X} & \mathbf{X} & \mathbf{X} \\ \mathbf{X} & \mathbf{X} & \mathbf{X} \\ \mathbf{X} & \mathbf{X} & \mathbf{X} \\ \mathbf{X} & \mathbf{X} & \end{array}$

$\mathbf{X}$

$\mathbf{X}$

$\mathbf{X}$
$\mathbf{X}$
$\mathbf{X}$
$\mathbf{X}$

$X^{*} \quad X$

$X^{*}$

$X^{*}$

$\mathrm{X}^{*}$

$\mathrm{X}^{*}$

$X^{*}$

$\mathrm{X}^{*}$

$\mathrm{X}^{*}$

$\mathrm{X}^{*}$

$\mathrm{X} \quad \mathrm{X} \quad \mathrm{X}$

$\mathrm{X} \quad \mathrm{X} \quad \mathrm{X}$

$\mathbf{X}$

$\mathbf{X}$

$\begin{array}{lll}\mathbf{X} & \mathbf{X} & \mathbf{X} \\ \mathbf{X} & \mathbf{X}\end{array}$

$\mathbf{X}$

*This statistic is to be included in Versions 7 and 8.

**These analyses also include repeated measures designs.

†No ANCOVA is computed. t+No MANCOVA is computed. 
Table 2

General Characteristics of Seven Integrated Packages

\begin{tabular}{|c|c|c|c|c|c|c|c|}
\hline \multirow[b]{2}{*}{ Characteristics } & \multicolumn{7}{|c|}{ Integrated Packages } \\
\hline & SPSS & DATA-TEXT & OSIRIS & OMNITAB & SAS & MANOVA & MULT \\
\hline Version & 6 & 3.2 & III & 5 & & II & $\mathbf{V}$ \\
\hline Date of Release & 1975 & 1975 & 1973 & 1970 & 1972 & 1974 & 1974 \\
\hline Language(s) & FORTRAN IV & FORTRAN IV & $\begin{array}{l}\text { FORTRAN IV } \\
\text { PL/1 }\end{array}$ & $\begin{array}{c}\text { ANSI } \\
\text { FORTRAN }\end{array}$ & $\begin{array}{c}\text { FORTRAN IV } \\
\text { Assembly } \\
\text { PL/1 }\end{array}$ & FORTRAN IV & FORTRAN IV \\
\hline Machine(s) & $\begin{array}{c}\text { IBM } 360 / 70 \\
\text { CDC } 6000 \\
\text { UNIVAC } 1100 \\
\text { Others* }\end{array}$ & $\begin{array}{c}\text { IBM } 360 / 70 \\
\text { CDC } 6000\end{array}$ & IBM $360 / 70$ & MI** & IBM 360/70 & IBM $360 / 70$ & $\begin{array}{c}\text { IBM } 360 / 70 \\
\text { CDC } 6000 \\
\text { UNIVAC } 1100\end{array}$ \\
\hline $\begin{array}{l}\text { Core Required } \\
\text { (K by tes) }\end{array}$ & $220 \mathrm{~K}$ & $250 \mathrm{~K}$ & $104 \mathrm{~K}$ & $90 \mathrm{~K}$ words & $120 \mathrm{~K}+$ & $125 \mathrm{~K}$ & $150 \mathrm{~K}+$ \\
\hline \multicolumn{8}{|l|}{$\operatorname{Cost}$} \\
\hline $\begin{array}{l}\text { Nonprofit } \\
\text { Profit } \\
\end{array}$ & $\begin{array}{l}\$ 1,250^{* * *} \\
\$ 5,000\end{array}$ & $\begin{array}{l}\$ 750^{\dagger} \\
\$ 1,000^{\circ}\end{array}$ & $\begin{array}{l}\$ 750 \\
\$ 1,000 \\
\end{array}$ & $\begin{array}{l}\$ 250 \\
\$ 250\end{array}$ & $\begin{array}{l}\$ 600+\dagger \\
\$ 1,000\end{array}$ & $\begin{array}{l}\text { N/A } \\
\text { N/A }\end{array}$ & $\begin{array}{l}\$ 840 \\
\$ 1,200\end{array}$ \\
\hline
\end{tabular}

Table 3

Availability and User's Manuals of Seven Integrated Packages

\begin{tabular}{lcc}
\hline \multicolumn{1}{c}{ Package } & Availability & User's Manual \\
\hline SPSS & National Opinion Research Center & Nie, N. H , Hull, C. H Jenkins, J. G., Steinbrenner
\end{tabular}

University of Chicago

6030 South Ellis Avenue

Chicago, Illinois 60637

(312) 753-1546

DATA-TEXT

Dr. David J. Armor

3625 Serra Road

Malibu, California 90265

(213) 456-3015

OSIRIS

Institute for Social Research

P.O. Box 1248

Ann Arbor, Michigan 48106

(313) 764-4417

OMNITAB

Computer Products Group

U. S. Department of Commerce

National Technical Information Service

5285 Port Royal Road

Springfield, Virginia 22151

(703) $321-7281$

SAS

SAS Project

Institute of Statistics

North Carolina State University

P.O. Box 5457

Raleigh, North Carolina 27607

(919) $737-2585$

MANOVA

Dr. Elliot M. Cramer

L. L. Thurstone Psychometric Laboratory

University of North Carolina

Chapel Hill, North Carolina 27514

(919) 933-5085

MULTIVARIANCE
International Educational Services

P.O. Box A3650

Chicago, Illinois 60690

(312) 684-4920 K., \& Bent, D. H. SPSS: Statistical package for the social sciences (2nd ed.). New York: McGrawHill, 1975.

Armor, D. J., \& Couch, A. S. DATA-TEXT primer. New York: Free Press, 1972.

Institute for Social Research. OSIRIS III, Volume 1: System and program description. Ann Arbor: University of Michigan, 1973.

Hogben, D., Peavy, S.T., \& Varner, R. N. OMNITAB II user's reference manual. (National Bureau of Standards Technical Note 552). Washington, D. C: U. S. Government Printing Office, 1970. (SD No. C13.46:552)

Service, J. A user's guide to the statistical analysis system (SAS). Raleigh: North Carolina State University, 1972.

Cramer, E. M. Revised MANOVA program. Chapel Hill: University of North Carolina, 1974.

Finn, J. D. MULTIVARIANCE: Univariate and multivariate analysis of variance, covariance, and regression. Chicago: National Educational Resources, 1972. 
and parametric statistics with the exceptions of ANCOVA and multivariate analysis. ANCOVA and certain applications of MANOVA could even be computed by the multiple regression program.

The SAS package possesses powerful parametric statistical features comparable to OSIRIS and the two specialized integrated packages. Its flexible least squares and randomized plans for experiment programs are not available in any of the other packages. SAS also incorporates most of the options offered by the largest multivariate package (MULTIVARIANCE) at a lower cost.

Nonparametric statistical analysis of ordinal data seems to be the single category conspicuously neglected in all of the packages. To compensate for this deficiency, researchers have written their own specific, machinedependent programs, as evidenced by the numerous program abstracts that have appeared over the past decade in journals such as Educational and Psychological Measurement, Behavioral Science, and Behavior Research Methods \& Instrumentation. There are very few nonparametric "packages" that are capable of handling a variety of research problems, for example, NPSTAT (Computer Institute for Social Science Research, 1970) and SIEGEL (Vegelius, 1975).

Additional information on the foregoing packages can be obtained by writing the distributors. The addresses and user's manuals for the seven packages are listed in Table 3.

\section{REFERENCE NOTES}

1. Hall, J. Computer software for survey analysis. Social Science Research Council Newsletter, Special Supplement, No. 20, October 1973.

2. Hall, J. Register of software for survey analysis. Social Science Research Council Newsletter, No. 25, August 1974.

3. Rowe, B., Hall, J., \& Scheer, M. Computer software for survey analysis. Social Science Research Council Newsletter, Special Supplement, No. 29, November 1975.

4. Tauchi, H. J. Abstracts of statistical computer programs.

IBM Statistical Program Evaluation Committee, 1965.

5. SPSS, Inc. SPSS Newsletter, No. 8, July 1975. Pp. 5-6.

\section{REFERENCES}

Allerbeck, K. R. Data analysis systems: A user's point of view. Social Science Information, 1973, 10, 23-35.

Anderson, R. E., \& Coover, E. R. Wrapping up the package: Critical thoughts on applications software for social data analysis. Computers and the Humanities, 1972, 7, 81-91.

ARMOR, D. J. Developments in data analysis systems for the social sciences. Social Science Information, 1970, 9. 145-156.

Clyde, D. J., Cramer, E. M., \& Sherin, R. J. Multivatiate statistical programs. Coral Gables, Fla: Biometric Laboratory, University of Miami, 1966.

Computer Institute for Social Science Research. NPSTAT, Non-parametric statistical system. East Lansing, Mich: Michigan State University, 1970.

Coover, E. R., Dyer, J. A., Gross, J. R., Johnson, C. C., LUtgen, G. L., \& Miner, J. F. Design of an optimally compatible social data analysis system: The first steps. Social Science Information, 1974, 13, 105-146.

Francis, I., Heiberger, R. M., \& Velleman, P. F. Criteria and considerations in the evaluation of statistical program packages. The American Statistician, 1975, 29, 52-56.

Schucany, W. R., Minton, P. D., \& Shannon, B. S., JR. A survey of statistical packages. Computing Surveys, 1972. $4,65-79$.

VEGELIUS, J. SIEGEL, A FORTRAN IV program for nonparametrical methods. Educational and Psychological Measurement, 1975, 35, 713-715.

\section{NOTE}

1. University professors, practitioners, graduate students, and about 60 textbooks served as the sources for the list of statistical procedures.

(Received for publication September 7, 1976; revision accepted October 29,1976 .) 